\title{
Hibiscus Sabdariffa Topical Extract Ointment Inhibits Inflammatory Signals and Exhibits Antibacterial Activity on the Rat Burn Injury: Emerging Approach for New Application in the Superficial Burn Care
}

Rania khalil ( $\square$ rania742002@yahoo.com )

Delta University for Science and Technology

Walied S Abdo

Kafrelsheikh University Faculty of Veterinary Medicine

Ghada S. El-Tanbouly

Delta University for Science and Technology

Dina Johar

Ain Shams University

Mahmoud Saad Abdel-Halim

Zagazig University

Sameh Saber

Delta University for Science and Technology

\section{Research Article}

Keywords: Hibiscus sabdariffa, TGF- $\beta$, TNF- $a$, TLRs, Wound healing, Pseudomonas aeruginosa

Posted Date: January 27th, 2022

DOI: https://doi.org/10.21203/rs.3.rs-1272012/v1

License: (c) (i) This work is licensed under a Creative Commons Attribution 4.0 International License.

Read Full License 


\section{Abstract}

Wound healing comprises organized events involving tissue repair and regeneration. The discovery of Toll-like receptors (TLRs) sheds recent light on the mechanisms involved in initiating inflammatory responses throughout the healing cascades. Hibiscus sabdariffa (HS) components may exhibit a wound healing action owing to their antioxidant and anti-inflammatory activities. This study designed to investigate the effect of HS loaded in an ointment base on the rat burn model compared to that of the standard Iruxolß ointment. A burn injury model was used to evaluate the wound healing potency of the preparation. Rats were treated with ointments three times through $24 \mathrm{hrs}$. Findings revealed that strong antioxidant property of the HS-loaded ointment augmented the skin healing potential by stimulating biomarkers required for skin regeneration. HS repressed the burning-induced inflammation by the effective reduction in the levels of tumor necrosis factor a (TNF-a) and IL-6 through TLR4 protein inhibition. Topical HS inhibited hypertrophic scarring by downregulating transforming growth factor-beta (TGF- $\beta$ ) levels. HS extract possesses a potential bactericidal activity against highly resistant clinical isolates of Pseudomonas aeruginosa. Altogether, study proclaims that HS-loaded topical preparations could be a valuable product that serve as adjuvants to accelerate burn wound healing through inactivating the TLR4 pathway.

\section{Introduction}

Wounds are physical injuries caused by opening or breaking of the skin. This skin injury causes disturbance in the anatomy and function, resulting in the discontinuity of the epithelium with or without the loss of the skin connective tissue. The process of wound healing comprises a cascade of biochemical and cellular events that include tissue repair and renewal. The stages of wound healing involve four processes: hemostasis, inflammation, proliferation, and maturation [1, 2].

In the inflammatory stage, impaired cells, bacteria, and pathogens are detached from the wound space. Nutrients, enzymes, white blood cells, and growth factors cause swelling, ache, and flush that are generally observed through this phase of wound healing. Inflammation, as a natural component of the healing process, is problematic only when it is persistent or excessive [3]. Inflammatory cytokines stimulate growth factors such as TGF- $\beta$ and a group of fibroblast growth factors, leading to infiltration of stimulated fibroblasts to the wounded area. Although an accurate level of cytokines is vital for healing, abnormal levels of proinflammatory cytokines lead to disproportionate fibroblast production, thereby initiating hypertrophic scarring, which lead to substantial deformity in skin tissue. Therefore, proinflammatory cytokines and growth factors play a pivotal part in the consequences of inflammation [4]. In spite of the substantial role of macrophages in wound healing, as they produce more proinflammatory cytokines (TNF-a) and interleukin (IL-6) in early wounds and less TGF- $\beta$, whereas the opposite is observed in later stage of wound healing [5].

All TLRs have an extremely homologous cytoplasmic toll/interleukin (IL)-1 receptor domain (TIR), a transmembrane domain, and an extracellular domain. TLR1, 2, 4, 5, 6, 10, and 11 locate on the exterior of 
the cell membrane, whereas TLR3, 7, 8, 9, 12, and 13 are extant on the membranes of intracellular compartments such as endoplasmic reticulum and lysosomes. TLR4 signaling is one of the vital mechanisms for inflammation $[5,6]$, and is a major receptor involved in different physiological circumstances, including wounds, shock, and autoimmunity. TLR4 has been explored as a target to inhibit interactions, deliver signaling molecules that initiate inflammatory responses, sustain epithelial cell integrity, and encourage recovery from injury [7]. Furthermore, new insights have shown that TLR4 stimulation enhances TGF- $\beta$ activity. Tissue damage caused by wounds leads to resident accumulation of endogenous TLR4 ligands named "damage-associated molecular patterns (DAMPs)" such as fibronectin-EDA and tenascin-C. DAMPs in sequence stimulate innate immune signaling in local fibroblasts via TLR4, resulting in augmented matrix and TGF- $\beta$ production [8].

There are two TLR signaling pathways: the MyD88-dependent pathway and the MyD88-independent pathway (The TIR domain-containing adapter-inducing interferon (IFN)- $\beta$-dependent pathway) [9]. The MyD88-dependent pathway caused the induction of NFK $\beta$ - dependent transcription of inflammatory cytokines such as TNF-a, IL-1, IL-6, IL-8, IL-12, and MIP2. The MyD88-independent pathway promotes the $\mathrm{Nf} K \beta$ and IFN regulatory factors, which in turn upregulate the inflammatory cytokines and IFN genes, respectively [10].

Wounds and burns are easily infected with microbes [11, 12]. Microbial infections of wounds slow down the healing process and may badly deteriorate to systemic infections. Topical antiseptics and antimicrobial therapy are crucial to control microbial colonization thus preventing the development of invasive infection [13]. $P$. aeruginosa is a leading cause of burn wound infection leading to fatal issues if not properly treated. $P$. aeruginosa is inherently resistant to most antibiotics $[14,15]$ which represents further challenge to manage burn wounds infected with such bacteria.

Hibiscus sabdariffa L. (roselle) (HS), belonging to the Malvaceae family, grows in several countries. This plant is commonly used in traditional remedies for its abundant content of phytochemicals such as polyphenols. HS is used in the treatment of several progressive diseases such as hyperlipidemia, hypertension, cancer and inflammatory diseases of the kidney and liver. HS active constituents are known to have a preventive effect against chronic and degenerative diseases associated with oxidative stress [16]. Its polyphenol content, particularly anthocyanins, may be responsible for its antioxidant capability. Among its anthocyanins, 85\% comprise delphinidin-3-sambubioside (Dp3-Sam), which is the major source of the antioxidant effect of roselle extract. Dp3-Sam is isolated from dried calices, could inhibit proliferation of human leukemia cells in vitro by inducing apoptosis, which was characterized by the activation of caspase-3, -8 , and -9 and cell morphology, DNA fragmentation, and inactivation of poly (ADP) ribose polymerase (PARP). In addition, recent studies show that anthocyanin aglycons, especially cyanidin (Cy) and delphinidin (Dp), could reverse the in vitro expression of inflammatory mediators such as prostaglandin (PG) E2 and cyclooxygenase (COX-2) [17]. The antibacterial potential of HS extracts was previously evaluated against broad spectrum of bacterial strains $[18,19]$, however it is necessary to test this activity against $P$. aeruginosa clinical isolates obtained from wound burn infection. 
Emergency of burn cases is crucial in the first few hours and would in fact account for morbidity and mortality. This study investigates whether roselle extract has the potential to perform as an active wound healing agent, and to test whether such potential is antioxidant, anti-inflammatory activities in in vivo model and evaluate the bactericidal activity against wound isolates of $P$. aeruginosa.

\section{Materials And Methods}

\subsection{Collection of plant and preparation of the extract}

Fresh flowers of HS obtained from Aswan cultivars, and then were seeded in the botanical garden of the Faculty of Pharmacy, Delta University for Science and Technology. Extraction was performed as described by [20]. Briefly, flowers were shade-dried at $25^{\circ} \mathrm{C}$, milled using a grinder, and sieved. Next, $372.58 \mathrm{~g}$ of fine flower powder were suspended in $1000 \mathrm{ml}$ of $70 \%$ ethyl alcohol for 48 hours at RT. The residue was removed by filtration (1-mm pore size), and then the extract was effectively concentrated using a rotary evaporator for $1 \mathrm{~h}$. The solid concentration was measured, then the extract was aliquoted and kept at $-20^{\circ} \mathrm{C}$ for further experiments.

\subsection{Qualitative phytochemical evaluation}

Different phytochemicals from flowers were extracted and labelled by different methods [21]. The main phytochemical groups in ethanol extracts of Hibiscus sabdariffa flowers were free amino acids, proteins, carbohydrates, gums, alkaloids, glycosides, fixed oil, phytosterols, phenolic compounds, mucilage, saponins, lignins, flavonoids and terpenoids.

\subsection{Chemicals and reagents}

Liquid paraffin and white soft paraffin were supplied from Pharmaceutics Department, Delta University for Science and Technology. Iruxol® ointment (1.2 IU, Smith nephew, Knoll Farmaceutica SpA, Muggio, Italy) was used as a standard ointment for comparing the wound healing potential of the plant extract. Analytical grade chemicals and reagents were used.

\subsection{Formulation of the ointment}

The ointment formulation $(10 \%(\mathrm{w} / \mathrm{w}))$ was prepared by infusing $10 \mathrm{~g}$ of the extract into $100 \mathrm{~g}$ of a simple ointment base (British Pharmacopoeia) [22]. It was prepared using a simple ointment base (liquid paraffin $(33.32 \mathrm{~g})$, white soft paraffin $(144.44 \mathrm{~g})$, to which $22.22 \mathrm{~g}$ of the extract was added. First, all the extract and liquid paraffin were supplemented and mixed with constant shaking in a water bath at $60^{\circ} \mathrm{C}$ to conquer a gel-like uniformity. White soft paraffin was allowed to melt, then the drug and liquid paraffin mixture were added portion-by-portion onto the melted white soft paraffin and mixed with constant stirring.

\subsection{Evaluation of ointment stability}


The freshly prepared cooled ointment was evaluated for color and appearance by visualization, and its consistency and homogeneity were also evaluated by rubbing within the fore and first fingers. Each ointment sample were located in a wide-mouthed plastic container and kept at $-5^{\circ} \mathrm{C}, 27^{\circ} \mathrm{C}(\mathrm{RT})$, and $40^{\circ} \mathrm{C}$. The homogeneity, color/appearance and consistency of these samples were checked daily for 14 days for any alterations. The $\mathrm{pH}$ values of the freshly prepared ointment were determined using a $\mathrm{pH}$ meter (accumet research AR10, Singapore). Ointment samples were put in storage at $27^{\circ} \mathrm{C}(\mathrm{RT})$, and their $\mathrm{pH}$ were checked daily for 14 days. Next, $5 \mathrm{~g}$ of each ointment sample were relocated into a $10-\mathrm{ml}$ beaker for experimentation.

\subsection{Testing for acute skin irritation and toxicity of the ointment}

The ointment was used for evaluating acute irritation and toxicity of the skin at $10 \% \mathrm{w} / \mathrm{w}$ according to the OECD guideline 402. The ointment was applied after shaving dorsal hair of rats. The treated region was checked for adverse dermal reactions such as erythema, irritation, inflammation, and itching compared to the control group treated with the ointment base but without the drug (placebo).

\subsection{Animals and experimental protocols}

Experimental protocols followed the ARRIVE guidelines of the National Centre for the Replacement, Refinement and Reduction of Animals in Research (NC3Rs). Adult male Wistar rats, with a typical weight of 200-250 g, were obtained from the animal facility at the faculty of pharmacy, Delta University (FPDU), and were accommodated in polypropylene cages with ad-lib access to basal diet and water. Animals allowed to acclimatize for one week.. Furthermore, all procedures were approved by the Institutional Animal Care and Use Committee at the FPDU. Rats were anesthetized with thiopental anesthesia (40 $\mathrm{mg} / \mathrm{kg}$ body weight) before commencing experimental wounds and surgical interventions were aseptically performed. Only healthy animals free from infections were used. Wound models were commonly used to evaluate the wound healing activity [23].

\subsection{Induction of burn}

Burn wounds were done as described by Cai et al. [24]. Briefly, the dorsal back of rats was smoothly shaved. A $100-\mathrm{g}$ cylindrical stainless steel rod $\left(1 \mathrm{~cm}\right.$-diameter) was heated to $100^{\circ} \mathrm{C}$ in boiling water. Contact burns were induced by placing the heated rod on the dorsum for $8 \mathrm{sec}$, the full $1 \mathrm{~cm}$-diameter wounded tissue was collected including epidermis, dermis and hypodermis, in which it was only one wound per animal. This procedure has been confirmed histologically to induce full-thickness injuries.

Animals were divided into four groups of six each as follows:

Group I: Normal topically applied with the ointment base (Negative control, placebo).

Group II: Burn-wounded topically applied with the ointment base (Positive control, placebo).

Group III: Wounded topically applied with the standard Iruxol® ointment. 
Group IV: Wounded topically applied with the prepared roselle extract ointment.

The respective ointments were applied to the entire wound area three times through $24 \mathrm{hrs}$.

\subsection{Collection of tissues}

Tissues from control and test groups were harvested after 24-hrs exposure to the wound and treatments. Tissues were washed in cold saline $(0.9 \% \mathrm{NaCl})$ to remove blood. The harvested cylinder-like tissue was cut longitudinally into two halves. One portion was fixed in $10 \%$ formalin for $24 \mathrm{~h}$ for histopathological examinations. The second half was stored at $-80^{\circ} \mathrm{C}$ until further determination of antioxidant and inflammatory markers. To determine antioxidant and inflammatory markers, the harvested skin tissue was homogenized in Tris Buffered Saline (TBS) [50 mM Tris-HCl: $150 \mathrm{mM} \mathrm{NaCl}, 1: 2]$ at pH 7.4 in UltraTurax homogenizer. The homogenates were then centrifuged at $4^{\circ} \mathrm{C}(5500 \mathrm{~g}, 15 \mathrm{~min})$.

\subsection{Assessment of burn intensity}

Burn intensity was assessed based on a collective arbitrary scale depending on the following aspects: blistering, swelling, redness, crust, secretion, and scar tissue formed. A score was given to each clinical feature when being established as follows: None, 0 ; mild, 1 ; moderate, 2; and severe, 3 . The scoring criteria were blindly visualized by a physician who is an experienced dermatologist in the field of skin care. The mean of a scoring criterion was calculated for each experimental group and added to the mean of every other individual scoring criterion to produce the total burn intensity value. In other words, the burn intensity value in a rat group is the total of the means of the scoring of every clinical feature as designated by the dermatologist.

\subsection{Histological and immunohistochemical analysis}

Standard histological technique was conducted. Tissue samples were stored in $10 \%$ formalin buffer solution, dehydrated, and then blocked with paraffin wax. Each blocked skin sample was cut into 5- $\mu \mathrm{m}-$ thick sections and stained with hematoxylin and eosin (H\&E) stain. The skin sections were checked under light microscope, and microphotographs were captured. Tissues obtained from the test group animals were also stained with Masson's trichrome, which is used to identify collagen deposition. Areas were quantified as area (A) \% using the Image J $1.52 \mathrm{n}$ software. The extent of collagen production was expressed as the \% of stained area relative to the total area. IL-6 immunolabeling was developed using IL6 antibodies (NB600-1131, Novus Biologicals, Littleton CO, USA). The immunoreactivity was indicated by calculating the immunopositive cells/ 1000 cell count.

\subsection{Determination of total antioxidant capacity}

Total antioxidant capacity (TAC) was determined using an enzymatic colorimetric method. All measurements followed the instructions of the manufacturer of the kit (Biodiagnostic, Egypt). The antioxidant capacity was determined based on the reaction of antioxidants in the sample with a defined 
amount of exogenously provided $\mathrm{H}_{2} \mathrm{O}_{2}$. The antioxidants present in the sample reduce a certain $\mathrm{H}_{2} \mathrm{O}_{2}$ amount. The remaining $\mathrm{H}_{2} \mathrm{O}_{2}$ is calorimetrically assayed in an enzymatic reaction that comprises the conversion of 3,5-dichloro-2-hydroxybenzenesulfonate into a colored product.

\subsection{Determination of inflammatory markers}

The protein concentrations of TNF- $\alpha$ and TGF- $\beta$ were quantified using ELISA according to the manufacturer's instructions (R\&D Systems, Minneapolis, MN, USA). Absorbance was read against blank in a Spectrum Max Plus 384 spectrophotometer (Sunnyvale, CA, USA), at dual wave lengths of $450 \mathrm{~nm}$ and $570 \mathrm{~nm}$. Cytokine's concentrations were calculated using standard curves obtained from recombinant cytokine activities.

\subsection{Western blot analysis for TLR4 expression}

Protein extraction from the skin tissues was performed using Radioimmunoprecipitation assay buffer (RIPA) (Bio Basic Inc., Markham Ontario, Canada). Protein concentrations were estimated using the Bradford method. For each lane, $20 \mu \mathrm{g}$ of protein was used and separated by $10 \%$ Sodium dodecyl sulphate-Polyacrylamide gel electrophoresis SDS-PAGE (Bio-Rad Laboratories, TNC, USA) and then transferred electrophoretically to pol-vinylidene fluoride (PVDF) membranes (Bio-Rad Laboratories, Inc.). The membranes were then incubated at RT for 2 hrs with a blocking solution consisting of $5 \%$ non-fat dried milk in TBST (10 mM Tris-Cl, $100 \mathrm{mM} \mathrm{NaCl}$, and 0.1\% Tween 20 (pH 7.5)). Next, the membranes were incubated overnight at $4^{\circ} \mathrm{C}$ with monoclonal antibodies specific for TLR4 and GAPDH and then with rabbit anti-rat secondary antibody conjugated to horseradish peroxidase (Thermo Fisher Scientific Inc., USA) at RT for $2 \mathrm{hrs}$. This was followed by washing four times with TBST at RT after each incubation. A chemiluminescent substrate (Clarity ${ }^{\text {TM }}$ Western ECL substrate, BIO-RAD, USA) was used to detect chemiluminescence according to the manufacturer's instructions. Then, the chemiluminescent signals were captured using a CCD camera-based imager, followed by image analysis to read the band intensity of the target proteins against the control sample after normalization by GAPDH on the Chemi Doc MP imager.

\subsection{Antibiotic sensitivity and evaluation of antibacterial activity}

The clinical isolates of $P$. aeruginosa used in this study were obtained from the collection bank of Microbiology and Immunology Lab, Faculty of Pharmacy, University of Zagazig. Bacterial isolates were recovered from human burn wound exudates and were purified and identified by morphology, pigmentation, Gram stain, biochemical tests, and growth on selective media. Antibiotic sensitivity was determined for the clinical isolates of $P$. aeruginosa by the disk diffusion method (DDM) [25] according to the Clinical and Laboratory Standard Institute. Bacterial culture media were purchased from purchased 
from Oxoid, (Hampshire, England), and Hi-Media, (India), antibiotic discs were obtained from Oxoid, (Hampshire, England) including Amikacin (AK, $30 \mu \mathrm{g})$, ampicillin (AM, $10 \mu \mathrm{g})$, ceftazidime (CAZ, $30 \mu \mathrm{g})$, cefoperazone (CFP, $75 \mu \mathrm{g})$, ciprofloxacin (CIP, $5 \mu \mathrm{g})$, gentamicin (CN, $10 \mu \mathrm{g})$, cefepime (FEP, $30 \mu \mathrm{g})$, gatifloxacin (GAT, $5 \mu \mathrm{g})$, and piperacillin/tazobactam (TPZ, $110 \mu \mathrm{g})$.

In vitro antibacterial activity of HS ethanolic extract was determined using cut-well agar diffusion [26]. Briefly, each bacterial isolate was grown in in $20 \mathrm{~mL}$ of tryptone soya broth medium (TSB) at $37 \circ \mathrm{C}$ for 2 days with constant shaking at $150 \mathrm{rpm} .100 \mathrm{uL}$ of $0.5 \mathrm{McF}$ arland turbidity standards $(1.0 \times 108 \mathrm{CFU} / \mathrm{mL})$ of each strain were streaked over a Mueller-Hinton agar plate using a cotton swab and wells of $6 \mathrm{~mm}$ were prepared. Then, $150 \mu \mathrm{L}$ HS ethanolic extract at a concentration of $250 \mathrm{mg} / \mathrm{mL}$ was loaded to the well. The plate was incubated for $24 \mathrm{~h}$ at $37^{\circ} \mathrm{C}$. The experiment was done in triplicates and the mean diameter of the zone of inhibition around the well was scored.

\subsection{Statistical analysis}

Data were expressed as the mean \pm standard deviation (SD). Statistical analysis was conducted using the GraphPad Prism version 6 (GraphPad Software Inc., La Jolla, CA, USA). Differences between groups were examined using one-way ANOVA, followed by Tukey's Kramer multiple comparison test. Scores of burn intensity were expressed as the median \pm interquartile range and were compared using KruskalWallis followed by Dunn's multiple comparison test.

\section{Results}

\subsection{Effect of HS on the intensity of 2nd degree burn wound}

The effect of HS on the intensity of 2nd degree burn is presented in Table 1 and Fig. 1. After $24 \mathrm{hrs}$ of exposure to damage, the burn group showed a significant elevation in the intensity of the burn compared with that of the normal unexposed group. The Iruxol-treated group showed that the intensity was significantly decreased to $40 \%$ compared to the burn group, whereas it was significantly increased only to $20 \%$ compared to the normal group. The HS-treated group showed that the intensity was significantly decreased by $50 \%$ compared to the burn group and was significantly increased to $30 \%$ compared with the normal group. 
Table 1

Clinical evaluation of burn skin lesions ( $N=6$ rats)

\begin{tabular}{|c|c|c|c|c|}
\hline & Normal skin & 2nd degree burn & Iruxol treatment & HS treatment \\
\hline \multicolumn{5}{|c|}{ Clinical features } \\
\hline Blistering & 0 & 1.83 & 0.50 & 1.00 \\
\hline Swelling & 0 & 1.83 & 0.33 & 0.83 \\
\hline Redness & 0 & 0.83 & 0.33 & 0.50 \\
\hline Crust & 0 & 0.67 & 0.50 & 0.50 \\
\hline Scar tissue & 0 & 0.83 & 0.33 & 0.17 \\
\hline Intensity & 0 & 5.99 & 1.99 & 3 \\
\hline
\end{tabular}

\subsection{Effect of HS on collagen deposition}

The effect of HS on collagen deposition is shown in Fig. 2. Collagen deposition significantly increased in the burn group compared with that of the normal group. Collagen deposition was significantly decreased in the Iruxol-treated group compared to burn group $(p<0.01)$. Additionally, the HS-treated group showed a significant decrease in collagen deposition compared to that of the burn group $(p<0.05)$.

\subsection{Histopathological examination of skin tissues}

The photomicrographs of the H\&E and the Masson's trichrome staining revealed normal epidermal layer formed of stratified epithelium with marked keratinization and normal dermal layer contained numerous hair follicles in normal skin group. The 2nd degree burn group exhibited minimal degree of epithelialization with subepithelial edema. However, treatment with either Iruxol or HS resulted in marked epithelialization degree and normal dermal hair follicle appendages. Masson's trichrome staining indicated that the normal dermal layer consisted primarily of parallel collagen. Burn group was associated with dermal collagen formation. In contrast, Iruxol or HS treatments displayed minimal dermal collagen formation and resulted in a marked epithelialization degree with normal dermal hair follicle appendages Fig. 3.

\subsection{Immunostaining of IL-6}

A marked expression of IL-6 within the dermal layer of the burn group as indicated upon calculation of the \% immunopositive cells/ 1000 counted cell was clearly displayed. While the treated groups with either Iruxol or HS showed a marked decrease in the expression of IL-6 Fig. 4.

\subsection{Effect of HS on total antioxidant capacity (TAC)}


The burn group showed a significant $(P<0.05)$ decrease in the level of TAC compared to the normal group. In the Iruxol-treated groups, there was a non-significant change in the levels of TAC compared to the burn group. However, in the HS-treated groups, there was a significant increase in the levels of TAC compared to the burn group $(P<0.05)$ Fig. 5.

\subsection{Effect of HS on TNF-a}

The burn group showed a significant increase in the levels of TNF- $a(P<0.05)$ compared to the normal group. The Iruxol- or HS-treated group of rats exhibited a significant decrease in TNF-a level $(P<0.05)$ compared to the burn group Fig. 6.

\subsection{Effect of HS on TGF- $\beta$}

The burn group showed a significant increased levels of TGF- $\beta$ compared to the normal group. The Iruxoltreated group showed non-significant change in the level of TGF- $\beta$ compared to that of the burn. However, the HS-treated group showed a significant decrease those levels compared to the burn group $(P<0.05)$ Fig. 7.

\subsection{Effect of HS on TLR4 expression in different groups}

The burn group showed a significant $(P<0.05)$ increase $(8-$ fold $)$ in the relative expression of TLR4 compared with that of the normal group. The Iruxol- or HS-treated groups showed that TLR4 expression was significantly decreased $(P<0.05)$ compared to the burn group. However, the HS-treated group exhibited a significant decrease $(P<0.05)$ in the expression of TLR4 compared to that of the Iruxoltreated group Fig. 8.

\subsection{Antibacterial activity of HS on burn wound isolates of $P$. aeruginosa}

We finally evaluated the bactericidal activity of HS extract against 2 clinical isolates of $P$. aeruginosa obtained from burn wound exudates. While the 2 isolates exhibited prominent resistance against different categories of antibiotics that target the bacterial cell wall, the bacterial ribosomes, or the bacterial DNA replicating enzymes, HS ethanolic extract at a concentration of $250 \mathrm{mg} / \mathrm{ml}$ clearly exhibits a bactericidal effect against wound isolates of $P$. aeruginosa giving zone of inhibition ranging from (24 \pm 5$)$ to (33 \pm 3.6) $\mathrm{mm}$ in diameter Fig. 9.

\section{Discussion}

Burn wound healing requires a well-organized cascade of biochemical and cellular events that involve tissue repair and regeneration. HS (roselle) is a widely used commercial traditional medicine, due to which it was primarily selected in our study. This study proposed that the content of polyphenols, especially anthocyanins, in roselle may be responsible for its antioxidant and anti-inflammatory properties essential for burn wound healing [27]. We intended to evaluate the effect of HS alcoholic extract loaded in an ointment base on healing based on its potential to regulate the link between TLR4 
and TGF- $\beta$ signaling pathways. Inhibition of these pathways may stimulate the antioxidant and antiinflammatory effects of HS which may be relevant to the observed skin regeneration in rats with superficial skin excision wounds.

Iruxol® is a topical collagenase preparation used for enzymatic debridement of necrotic tissue caused by ulcers and burns, and hence it was used for the purpose of comparison as a standard treatment in this study. Iruxol® contains the collagenase proteolytic enzyme derived from Clostridium histolyticum. Collagenase digests denatured collagen, cleanses necrotic tissues, and destroys the strands of endogenous collagen, which attach the necrotic residues to the wound bed. Using Iruxol $\circledast$ in uncomplicated wounds is considered to be well tolerated [28]. In the present study, after $24 \mathrm{hrs}$ of Iruxol® treatment, the superficial burns exhibited dramatic healing percentages (40\%) with the absence of charring and eschar due to its collagenase activity, which corresponded to the percentage of collagen deposition as evaluated using the Image $\mathrm{J}$ software.

In the case of HS ointment, healing occurred significantly but at a lower degree than that of Iruxol® treatment. This may be due to the autolytic debridement caused by topically applied natural products such as roselle. Our findings converge with the autolytic debridement with support from topically applied natural products such as honey, which is believed to go in the same way of the self-healing process to cleanse the wound from tissue debris. The healing activity of honey is attributed to its acidity, suppressing destructive proteases, and osmotic effect on the wound bed [29].

A pivotal crosstalk has been recognized between TLR4 and TGF- $\beta$ signaling cascades and wound healing. Till now, HS is the first suggestion to be used an inhibitor for both TLR4, TGF- $\beta$ and collagen deposition upon tissue damage. Hence, it is recognized that oxidative stress has serious role in the origin and development of wounds. Burning wounds in rats are in close proximity to human wound injuries in terms of histopathological features, characterized by a minimal degree of epithelialization, which significantly contributes to the progression of subepithelial edema through the production of dermal collagen [30]. Moreover, tissue injury correlates with strong local immune response that is connected to augmented production of cytokines, particularly TNF-a that can trigger oxidative burst. Effects of both Iruxol ${ }^{\circledR}$ and $\mathrm{HS}$ on burn wound healing were confirmed by histopathological examination, which revealed marked epithelialization degree, normal dermal hair follicle appendages and minimal dermal collagen formation in both groups compared with these in burn untreated group.

Emerging evidence indicates that TLR4 participates in the stimulation of innate immune response by triggering signaling cascades along with the release of several inflammatory cytokines such as TNF-a. Here, we found that HS ointment downregulates TNF- $a$ to $<50 \mathrm{pg} / \mathrm{ml}$, as the normal skin TNF-a level is $<50 \mathrm{pg} / \mathrm{mL}$ [31]. Comparison between Iruxol $\circledast$ and HS treatment results showed that HS treatment resulted in less tissue TNF-a levels than those obtained with Iruxol ${ }^{\circledR}$ ointment treatment, as the latter value was $>50 \mathrm{pg} / \mathrm{ml}$. This implied that roselle extract has an anti-inflammatory effect that helps in burn healing. A previous study reported that the levels of IL-6, nitric oxide (NO), and TNF-a in inflammatory cells could be significantly reduced by HS treatment. 
These inflammatory cytokines lead to the initiation of growth factors release leading to the infiltration of stimulated fibroblasts to the wounded area. Although the correct level of each cytokine is important for healing, abnormal levels of proinflammatory cytokines lead to extreme fibroblast proliferation, resulting in hypertrophic scarring, which lead to a robust scarring [32].

In this study, TGF- $\beta$ level was found to significantly decrease after 24 hrs of application of either Iruxol $\AA$ or HS ointments. Previously, studies reported that new tissue development and scar formation are two crucial steps in the healing process. Fibroblasts are the primary cells in the healing process since they migrate to the wound where they proliferate. Then, these cells produce the extracellular collagen matrix. The functions of fibroblasts are regulated by growth factors [33]. Recently, there has been a focus on the double-edged weapon of TGF- $\beta$ level through hypertrophic scarring, which is a common occurrence in burn injuries, with an incidence of 70\% [34]. Moreover, the importance of TGF- $\beta$ level in keeping the vascular activity has been established in several studies, which have shown that increased TGF- $\beta$ level and its receptors may impair vessels formation [35].

However, the present data justified that HS has a greater antioxidant effect than Iruxol® ointment when topically applied three times/day. Numerous in vitro and in vivo reports confirmed that extracts of roselle possess actual antioxidant potential [36]. Wound progression is concomitant with oxidative stress that arises because of the disturbance in the balance between ROS production and endogenous antioxidant protection mechanism, which disturbs the biomolecules functioning in a reversible or irreversible manner [37]. It was established that ordinary cellular antioxidants such as superoxide dismutase and catalase participate in oxygen-protection responses in the tissue matrix by producing water and molecular dioxygen from superoxide. Increasing glutathione level in the tissue matrix results in enhanced glutathione peroxidase which by turn reduces peroxides [38]. The present study results indicate a significant increase in TAC in the HS-ointment-applied group as evidenced by the neutralization of additional free radicals in the wound, owing to the phenolic nature of HS, which ultimately encourage a synergistic outcome in wound healing [39].

It has been described that such effects were addressed for both alcoholic and aqueous HS extracts of flowers, seeds, or leaves through shielding effect against tert-butyl hydroperoxide (t-BHP)-induced oxidative damage. This activity of HS is due to the inhibition of xanthine oxidase activity [40], cytoprotection from impairment by lipid peroxidation, inhibition of Cu2+-mediated oxidation of LDL and the creation of thio-barbituric acid reactive substances (TBARs), maintaining the formation of malondialdehyde content, reduction of GSH exhaustion, and the increase in SOD and CAT activities [41].

Microbial contamination of wounds and burns greatly slows down the recovery, interferes with the healing process, complicates the management, and may lead to invasive infection. $P$. aeruginosa is a gram- negative bacterium that is commonly infecting wounds and burns leading to invasive burn sepsis which requires prompt therapeutic intervention [42]. One of the key aspects of burn care guidelines is to prevent infection and colonization of $P$. aeruginosa, however this pathogen is rapidly developing antimicrobial resistance against most used antibiotics. Broad spectrum antibacterial activity of HS 
ethanolic extract was previously addressed [43], but our study demonstrated a potential bactericidal activity of HS extract in tolerable concentrations against burn wound isolates of $P$. aeruginosa that exhibit multidrug resistance against different categories of antibiotics. Taken together, HS extract exhibits a dual role in wound healing through the anti-inflammatory activity which is necessary for the skin regeneration and the antibacterial effect which prevents microbial colonization and invasive infection.

\section{Conclusions}

HS ointment has an important role in healing wounds. HS extract enhanced the skin healing capacity by accelerating the healing process itself and stimulating the levels of skin regeneration biomarkers through its strong antioxidant and anti-inflammatory effects. Moreover, HS topical treatment significantly reduced hypertrophic scarring through its effect against TGF- $\beta$ levels. Therefore, it would be encouraging to discover natural medications that have the possibility to control the link between these TLR4 and TGF- $\beta$ signaling pathways. In addition, HS extract has a remarkable bactericidal activity against wound pathogens. Altogether, this study suggests that HS is a valuable extract to be used in cosmetics and alternative medicine, which may also be considered for use in combination with Iruxol ${ }^{\circledR}$ as a synergistic product to accelerate wound healing.

\section{Declarations}

\section{Authors' contributions:}

Rania M. Khalil contributed to conception, data acquisition and analysis, manuscript synthesis. Galal Yahya and Mahmoud S. Abdel-Halim performed the microbiology experiments, collected and wrote the data interpretation. Dina Johar and Ghada S. El-Tanbouly contributed to data analysis and discussion, manuscript synthesis. Walied S Abdo conducted the histological examinations, Western blot analysis, image analysis, data validation. Sameh Saber handled animals, provided software, conducted statistical analysis. All authors have read and approved the manuscript for publication.

\section{Data availability:}

The data obtained after analysis in the current study are available from the relevant authors upon reasonable request.

\section{Funding:}

This research did not receive any specific grant from funding agencies in the public, commercial, or notfor-profit sectors.

\section{Ethics approval and consent to participate:}

The study was conducted according to the ARRIVE guidelines of the National Centre for the Replacement, Refinement and Reduction of Animals in Research (NC3Rs). All procedures were permitted by the 
Institutional Animal Care and Use Committee at the Faculty of Pharmacy, Delta University for Science and Technology, Gamasa, Egypt.

\section{Consent for publication:}

Not applicable.

\section{Acknowledgments:}

Authors appreciate the support and encouragement provided by members of Delta University students (Esraa E. Borham, Laila A.Ibrahim, Mirvat E. EL shnawye, Hager A. Elrefaei, Asmaa M. Elsayed, Khloud T. Hammad, Amany T. Ali, Nesreen A. Elsaid, Zeinab E. Rizk, and Eman A. Elsaid) who were helpful in this project.

\section{Statements and Declarations:}

The authors declared that they have no conflicts of interest to this work. We declare that we do not have any commercial or associative interest that represents a conflict of interest in connection with the work submitted.

\section{References}

1. El-Gizawy, S.A., et al., Deferoxamine-loaded transfersomes accelerates healing of pressure ulcers in streptozotocin-induced diabetic rats. Journal of Drug Delivery Science and Technology, 2020. 58: p. 101732.

2. Yadav, E., et al., Antioxidant and anti-inflammatory properties of Prosopis cineraria based phenolic rich ointment in wound healing. Biomedicine \& Pharmacotherapy, 2018. 108: p. 1572-1583.

3. Ezzat, S.M., M.A. Choucry, and Z.A. Kandil, Antibacterial, antioxidant, and topical anti-inflammatory activities of Bergia ammannioides: A wound-healing plant. Pharmaceutical Biology, 2016. 54(2): p. 215-224.

4. Khalil, R., et al., Vildagliptin, a DPP-4 inhibitor, attenuates carbon tetrachloride-induced liver fibrosis by targeting ERK1/2, p38a, and NF-KB signaling. Toxicology and Applied Pharmacology, 2020. 407: p. 115246.

5. Portou, M.J., et al., The innate immune system, toll-like receptors and dermal wound healing: $A$ review. Vascular Pharmacology, 2015. 71: p. 31-36.

6. Youssef, M.E., et al., Interference With the AMPKa/mTOR/NLRP3 Signaling and the IL-23/IL-17 Axis Effectively Protects Against the Dextran Sulfate Sodium Intoxication in Rats: A New Paradigm in Empagliflozin and Metformin Reprofiling for the Management of Ulcerative Colitis. Frontiers in pharmacology, 2021. 12: p. 719984-719984.

7. Lafyatis, R. and A. Farina, New insights into the mechanisms of innate immune receptor signalling in fibrosis. The open rheumatology journal, 2012. 6: p. 72-79. 
8. Bhattacharyya, S., et al., Toll-Like Receptor-4 Signaling Drives Persistent Fibroblast Activation and Prevents Fibrosis Resolution in Scleroderma. Advances in wound care, 2017. 6(10): p. 356-369.

9. Saber, S., et al., The Supportive Role of NSC328382, a P2X7R Antagonist, in Enhancing the Inhibitory Effect of CRID3 on NLRP3 Inflammasome Activation in Rats with Dextran Sodium Sulfate-Induced Colitis. Journal of inflammation research, 2021. 14: p. 3443-3463.

10. Chen, L. and L.A. DiPietro, Toll-Like Receptor Function in Acute Wounds. Advances in wound care, 2017. 6(10): p. 344-355.

11. Coban, Y.K., Infection control in severely burned patients. World journal of critical care medicine, 2012. 1(4): p. 94-101.

12. Palmieri, T.L. and D.G. Greenhalgh, Topical Treatment of Pediatric Patients with Burns. American Journal of Clinical Dermatology, 2002. 3(8): p. 529-534.

13. Momtaz, S., et al., Wound healing activity of the flowers of Lilium candidum L. in burn wound model in rats. jmpir, 2020. 19(73): p. 109-118.

14. Gonzalez Manuel, R., et al., Effect of Human Burn Wound Exudate on Pseudomonas aeruginosa Virulence. mSphere. 1(2): p. e00111-15.

15. Pang, Z., et al., Antibiotic resistance in Pseudomonas aeruginosa: mechanisms and alternative therapeutic strategies. Biotechnology Advances, 2019. 37(1): p. 177-192.

16. Oboh, G., et al., Phenolic Constituents and Inhibitory Effects of Hibiscus sabdariffa L. (Sorrel) Calyx on Cholinergic, Monoaminergic, and Purinergic Enzyme Activities. J Diet Suppl, 2018. 15(6): p. 910922.

17. Jabeur, I., et al., Exploring the chemical and bioactive properties of Hibiscus sabdariffa L. calyces from Guinea-Bissau (West Africa). Food Funct, 2019. 10(4): p. 2234-2243.

18. Abdallah, E.M., Antibacterial efficiency of the Sudanese Roselle (Hibiscus sabdariffa L.), a famous beverage from Sudanese folk medicine. Journal of intercultural ethnopharmacology, 2016. 5(2): p. 186-190.

19. Abdallah, E.M., Antibacterial activity of Hibiscus sabdariffa L. calyces against hospital isolates of multidrug resistant Acinetobacter baumannii. Journal of Acute Disease, 2016. 5(6): p. 512-516.

20. Bhaskar, A. and V. Nithya, Evaluation of the wound-healing activity of Hibiscus rosa sinensis $L$ (Malvaceae) in Wistar albino rats. Indian journal of pharmacology, 2012. 44(6): p. 694-698.

21. Cranwell, P.B.H.L.M.M.C.J., Experimental organic chemistry. 2017.

22. Kodym, A. and T. Bujak, Physicochemical and microbiological properties as well as stability of ointments containing aloe extract (Aloe arborescens Mill.) or aloe extract associated to neomycin sulphate. Pharmazie, 2002. 57(12): p. 834-7.

23. Fikru, A., et al., Evaluation of in vivo wound healing activity of methanol extract of Achyranthes aspera L. J Ethnopharmacol, 2012. 143(2): p. 469-74.

24. Cai, E.Z., et al., Creation of consistent burn wounds: a rat model. Arch Plast Surg, 2014. 41(4): p. 31724. 
25. El-Baz, A.M., et al., The Link between Occurrence of Class I Integron and Acquired Aminoglycoside Resistance in Clinical MRSA Isolates. 2021. 10(5): p. 488.

26. Yahya, G., et al., Soil-Associated Bacillus Species: A Reservoir of Bioactive Compounds with Potential Therapeutic Activity against Human Pathogens. 2021. 9(6): p. 1131.

27. Riaz, G. and R. Chopra, A review on phytochemistry and therapeutic uses of Hibiscus sabdariffa L. Biomed Pharmacother, 2018. 102: p. 575-586.

28. Patry, J. and V. Blanchette, Enzymatic debridement with collagenase in wounds and ulcers: a systematic review and meta-analysis. Int Wound J, 2017. 14(6): p. 1055-1065.

29. Gilligan, A.M., et al., Comparative Effectiveness of Clostridial Collagenase Ointment to Medicinal Honey for Treatment of Pressure Ulcers. Adv Wound Care (New Rochelle), 2017. 6(4): p. 125-134.

30. Zielins, E.R., et al., Emerging drugs for the treatment of wound healing. Expert Opin Emerg Drugs, 2015. 20(2): p. 235-46.

31. Wang, J., et al., In vitro anti-inflammatory mechanism of Folium Hibisci Mutabilis leaves ethanol extracts. Afr J Tradit Complement Altern Med, 2014. 11(1): p. 127-130.

32. Shah, A. and S. Amini-Nik, The Role of Phytochemicals in the Inflammatory Phase of Wound Healing. Int J Mol Sci, 2017. 18(5).

33. Finnerty, C.C., et al., Hypertrophic scarring: the greatest unmet challenge after burn injury. Lancet, 2016. 388(10052): p. 1427-1436.

34. Larsson, J., et al., Abnormal angiogenesis but intact hematopoietic potential in TGF-beta type I receptor-deficient mice. Embo j, 2001. 20(7): p. 1663-73.

35. Kim, S.I., et al., Transforming growth factor-beta (TGF-beta1) activates TAK1 via TAB1-mediated autophosphorylation, independent of TGF-beta receptor kinase activity in mesangial cells. J Biol Chem, 2009. 284(33): p. 22285-22296.

36. Da-Costa-Rocha, I., et al., Hibiscus sabdariffa L. - a phytochemical and pharmacological review. Food Chem, 2014. 165: p. 424-43.

37. Süntar, I., et al., Wound healing and antioxidant properties: do they coexist in plants? Free Radicals and Antioxidants, 2012. 2(2): p. 1-7.

38. Frank, T., et al., Consumption of Hibiscus sabdariffa L. aqueous extract and its impact on systemic antioxidant potential in healthy subjects. J Sci Food Agric, 2012. 92(10): p. 2207-18.

39. Peng, C.H., et al., Hibiscus sabdariffa polyphenolic extract inhibits hyperglycemia, hyperlipidemia, and glycation-oxidative stress while improving insulin resistance. J Agric Food Chem, 2011. 59(18): p. 9901-9.

40. Bakhtiari, E., A. Hosseini, and S.H. Mousavi, Protective effect of Hibiscus sabdariffa against serum/glucose deprivation-induced PC12 cells injury. Avicenna J Phytomed, 2015. 5(3): p. 231-7.

41. Guardiola, S. and N. Mach, [Therapeutic potential of Hibiscus sabdariffa: a review of the scientific evidence]. Endocrinol Nutr, 2014. 61(5): p. 274-95. 
42. Bielecki, P., et al., Towards understanding Pseudomonas aeruginosa burn wound infections by profiling gene expression. Biotechnol Lett, 2008. 30(5): p. 777-90.

43. Márquez-Rodríguez, A.S., et al., In Vitro Antibacterial Activity of Hibiscus sabdariffa L. Phenolic Extract and Its In Situ Application on Shelf-Life of Beef Meat. Foods, 2020. 9(8).

\section{Figures}

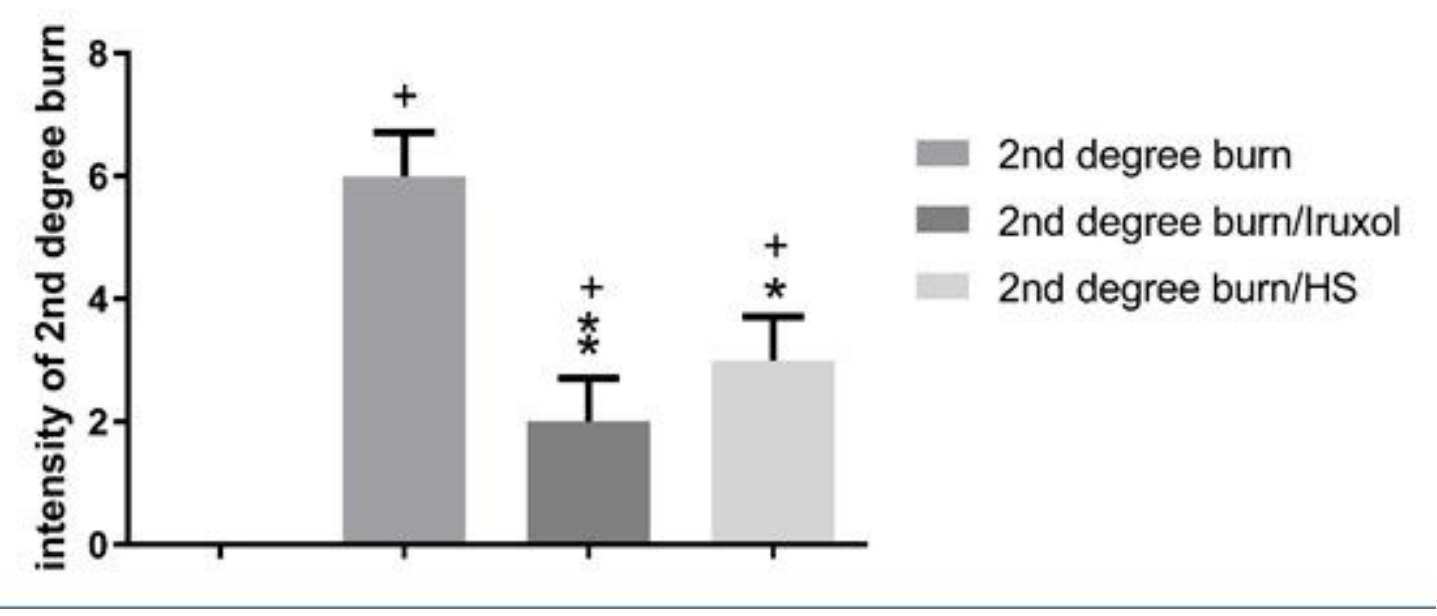

Figure 1

\section{Intensity of 2nd degree burn in different groups}

HS: Hibiscus sabdariffa,,$+ \mathrm{P}<0.05$ vs normal group; ${ }^{*} \mathrm{P}<0.05$ vs 2 nd degree burn group; $* *, \mathrm{P}<0.01$ vs 2nd degree burn group. Differences between groups were examined by Kruskal-Wallis followed by Dunn's multiple comparison test. $n=6$ 


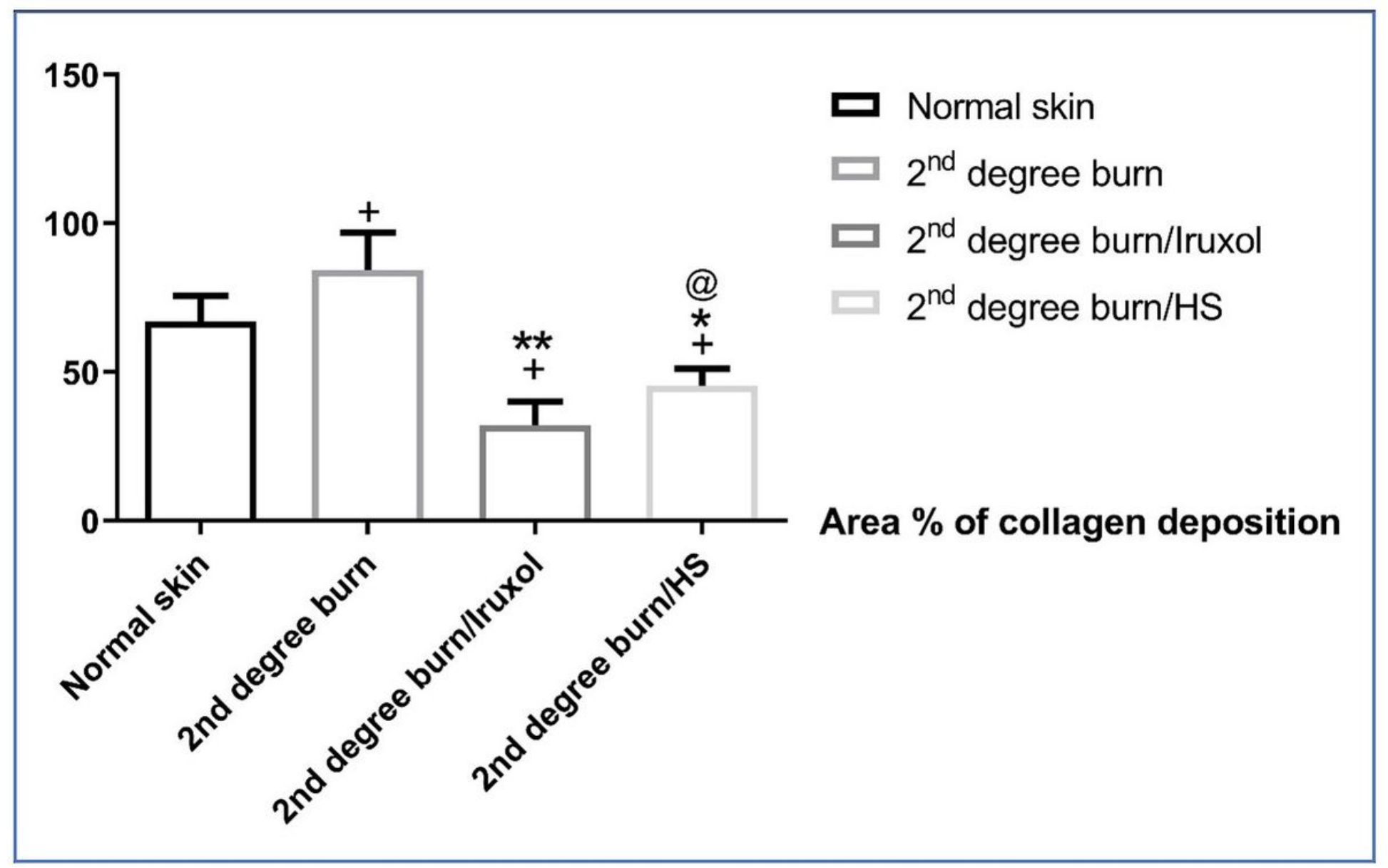

Figure 2

Percentage of collagen deposition

HS: Hibiscus sabdariffa,,$+(p<0.05)$ vs normal group; *, $(p<0.05)$ vs 2 nd degree burn group; $* *,(p<$ $0.01)$ vs 2 nd degree burn group; $@$, $(p<0.05)$ vs 2 nd degree burn/Iruxol-treated group. Differences between groups were examined by ANOVA/Tukey's Kramer multiple comparison test. $n=6$ 

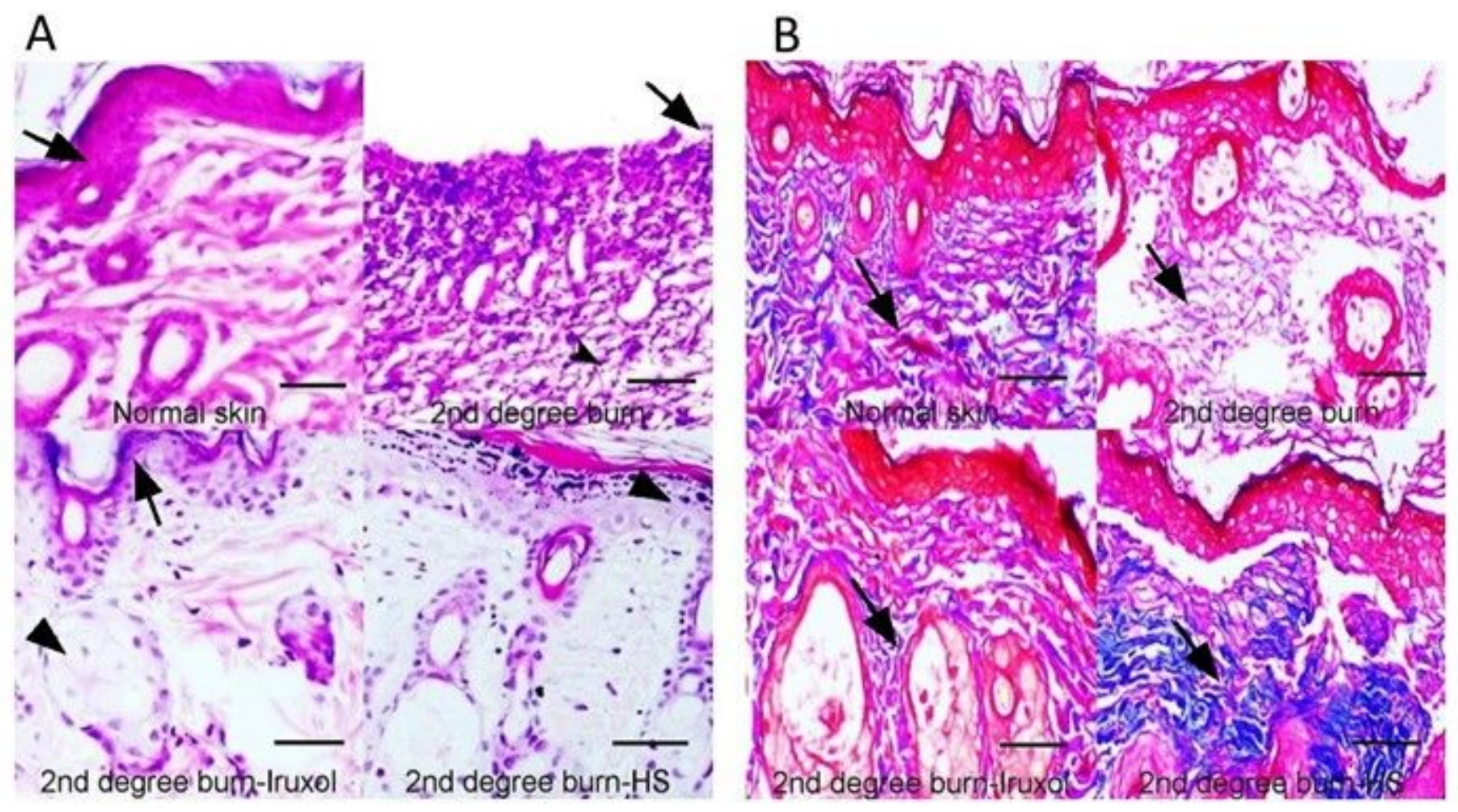

\section{Figure 3}

\section{Histopathological examination of skin tissues from different groups by H\&E staining}

The left panel (A) shows histopathological examination of skin tissues from different groups by H\&E staining, $\times 200$. Normal epidermal layer formed of stratified epithelium with marked keratinization and normal dermal layer, contained numerous hair follicles in the normal skin group (arrow). The 2nd degree burn group exhibited minimal degree of epithelialization (arrow) with subepithelial edema (arrowhead). In contrast, treatment with Iruxol resulted in marked epithelialization degree (arrow) and normal dermal hair follicle appendages (arrowhead). Treatment with HS resulted in marked epithelialization (arrowhead). Bar $=100 \mu \mathrm{m}$. The right panel (B) illustrates the Masson's trichrome staining, $\times 200$. Masson's trichrome staining indicated normal dermal layer of primary parallel collagen (arrow). The 2 nd degree burn group was associated with massive dermal collagen formation (arrow). In contrast, treatment with Iruxol resulted in minimal dermal collagen formation (arrow). Treatment with HS resulted in minimal dermal collagen formation. Bar $=100 \mu \mathrm{m}$. 

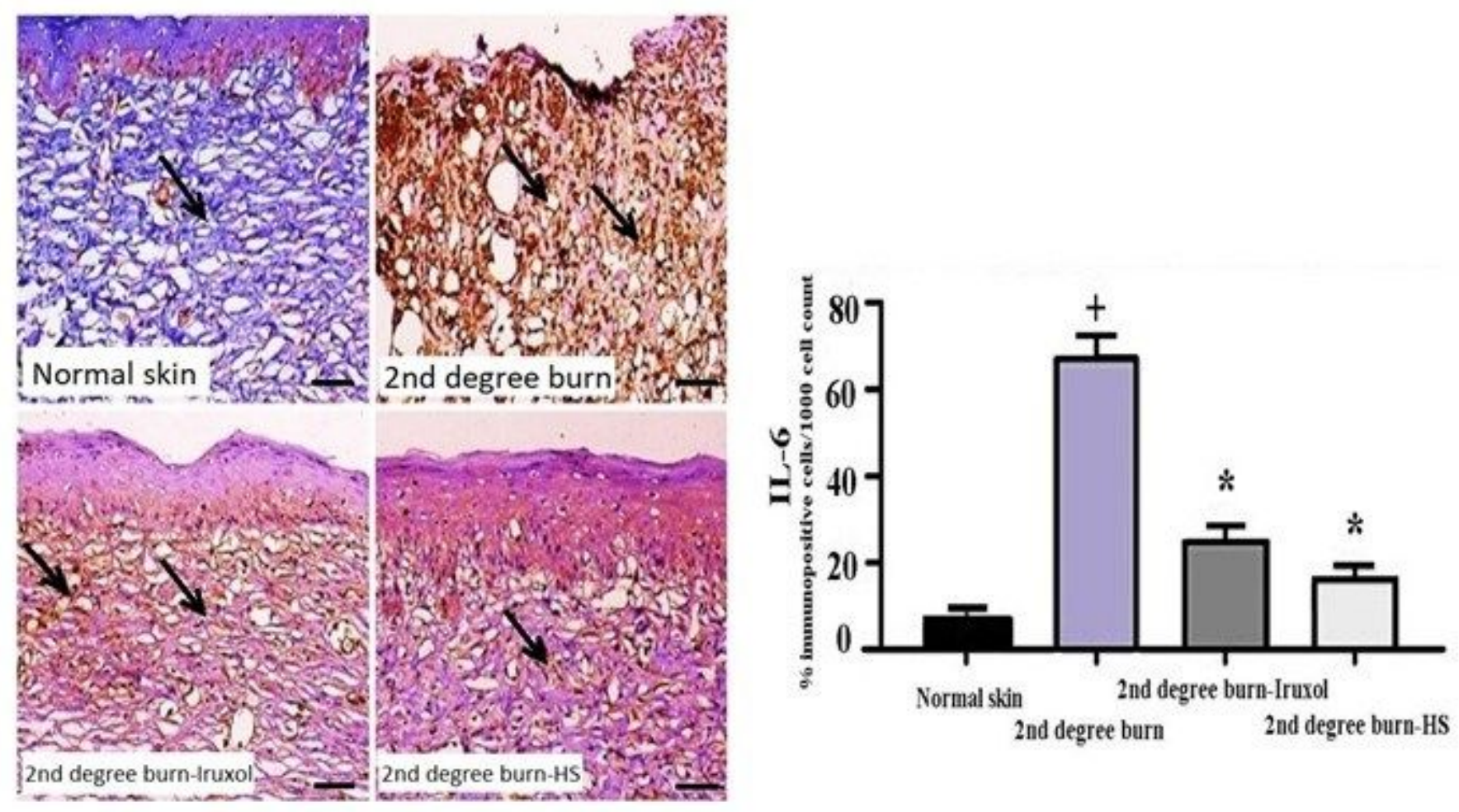

Figure 4

Immunohistochemical detection of IL-6 in skin tissues

Immunohistochemical detection of IL-6 in skin tissues, $\times 200$. Arrows indicate expression of IL- 6 within the dermal layers of different groups. Marked immunolabeling was detected in the 2 nd degree burn group of rats, while decreased expression was detected in the treatment groups. Bar $=100 \mu \mathrm{m} .+, \mathrm{P}<0.05 \mathrm{vs}$ normal group; *, $\mathrm{P}<0.05$ vs 2 nd degree burn group.

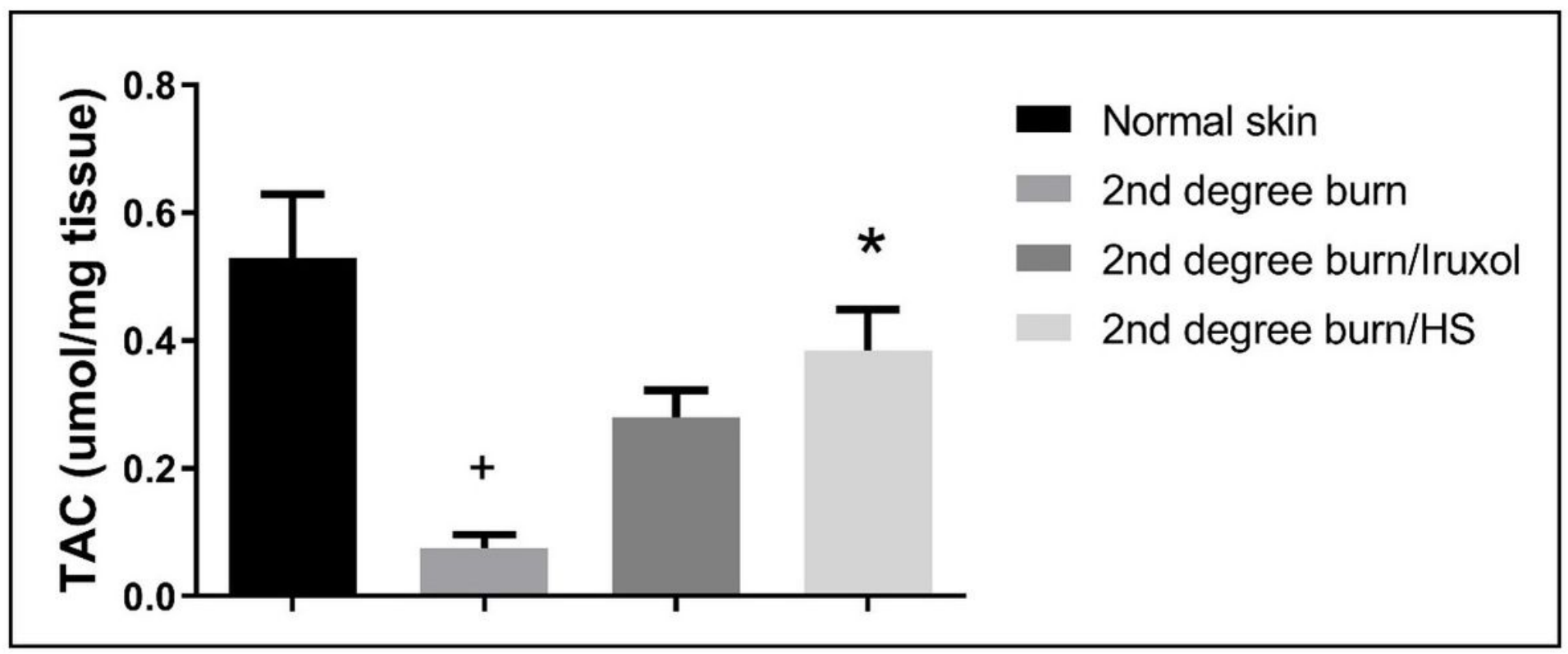


Figure 5

\section{Effect of HS on total antioxidant capacity (TAC)}

TAC: Total antioxidant capacity; HS: Hibiscus sabdariffa;,$+(P<0.05)$ vs normal group; *, $(P<0.05)$ vs 2nd degree burn group. Differences between groups were examined by ANOVA/Tukey's Kramer multiple comparison test. $n=6$

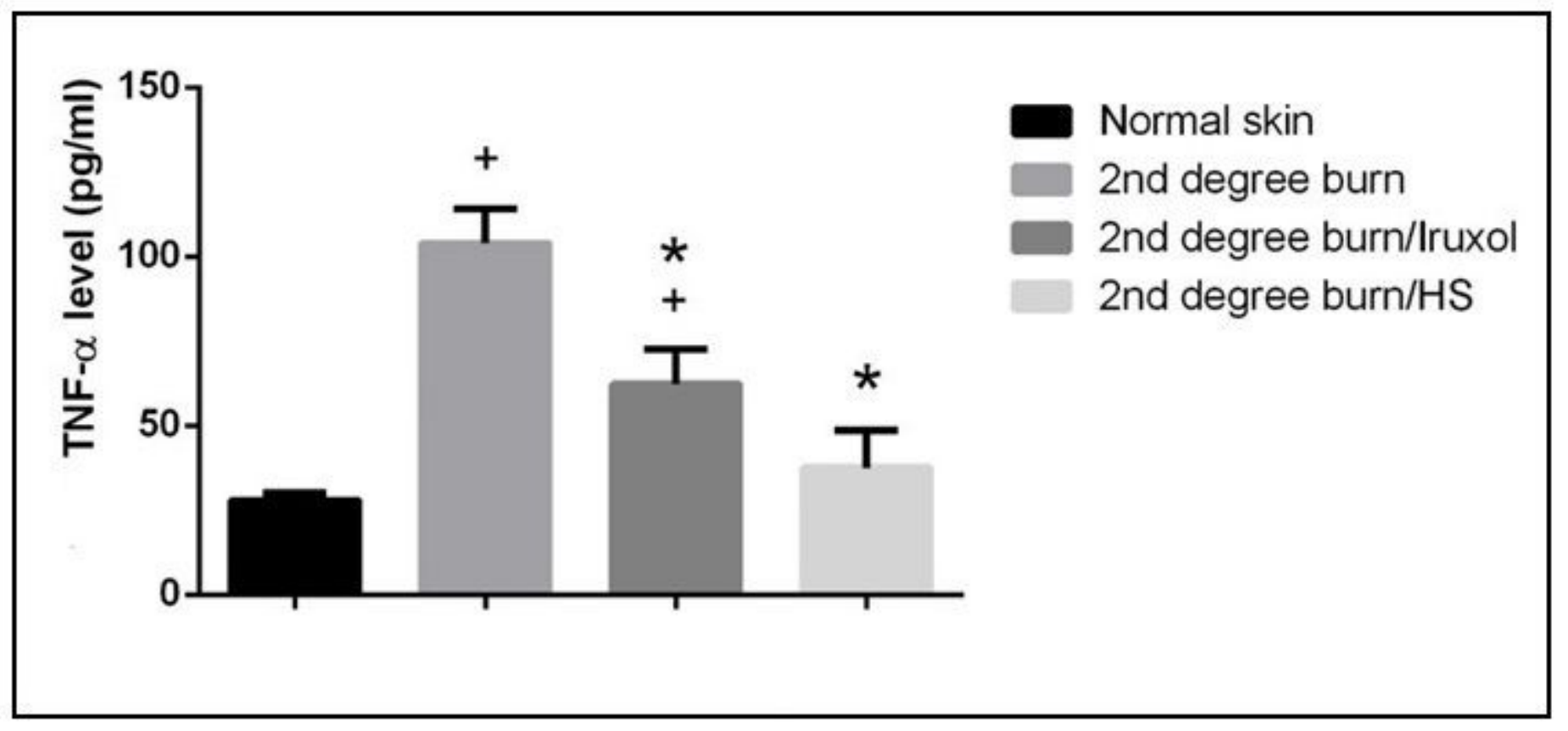

Figure 6

\section{Effect of HS on TNF-a}

TNF-a: Tumor necrosis factor alpha; HS: Hibiscus sabdariffa;,$+(P<0.05)$ vs normal group; ${ }^{*},(P<0.05)$ vs 2 nd degree burn group. Differences between groups were examined by ANOVA/Tukey's Kramer multiple comparison test. $\mathrm{n}=6$ 


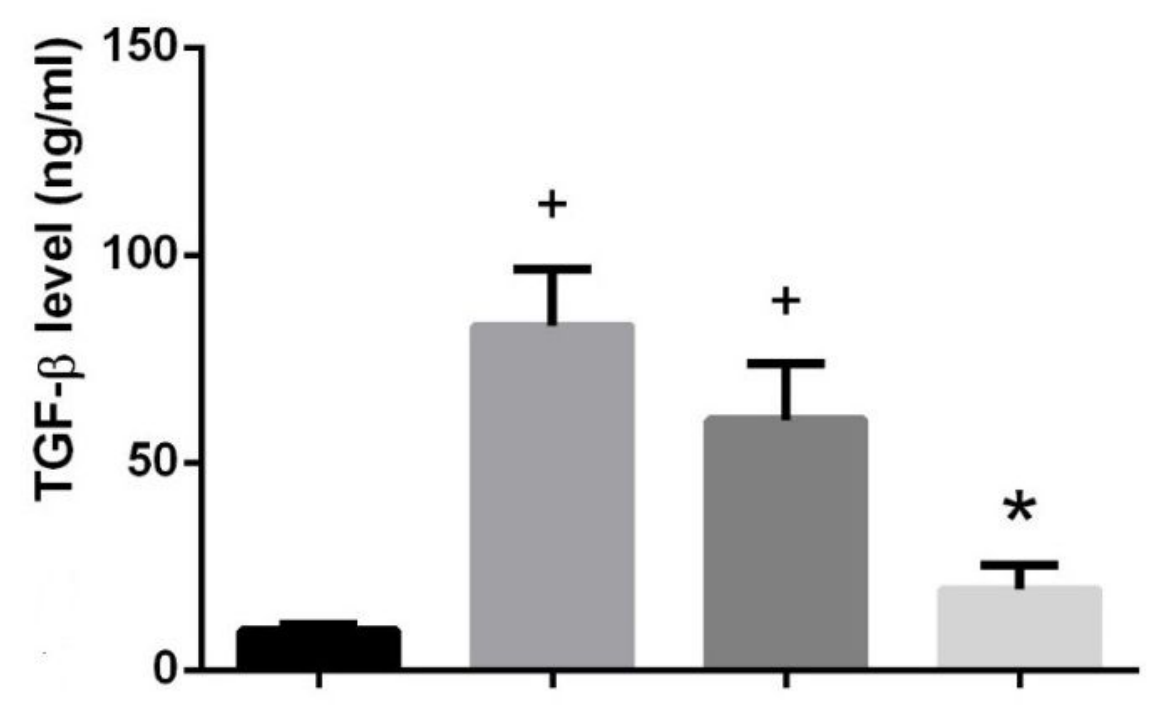

Normal skin

2nd degree burn 2nd degree burn/lruxol 2nd degree burn/HS

Figure 7

Effect of HS on transforming growth factor $\beta$ (TGF- $\beta$ ) level

TGF- $\beta$ : transforming growth factor-beta; HS: Hibiscus sabdariffa;,$+(\mathrm{P}<0.05)$ vs normal group; ${ }^{*},(\mathrm{P}<$ $0.05)$ vs 2 nd degree burn group. Differences between groups were examined by ANOVA/Tukey's Kramer multiple comparison test. $\mathrm{n}=6$

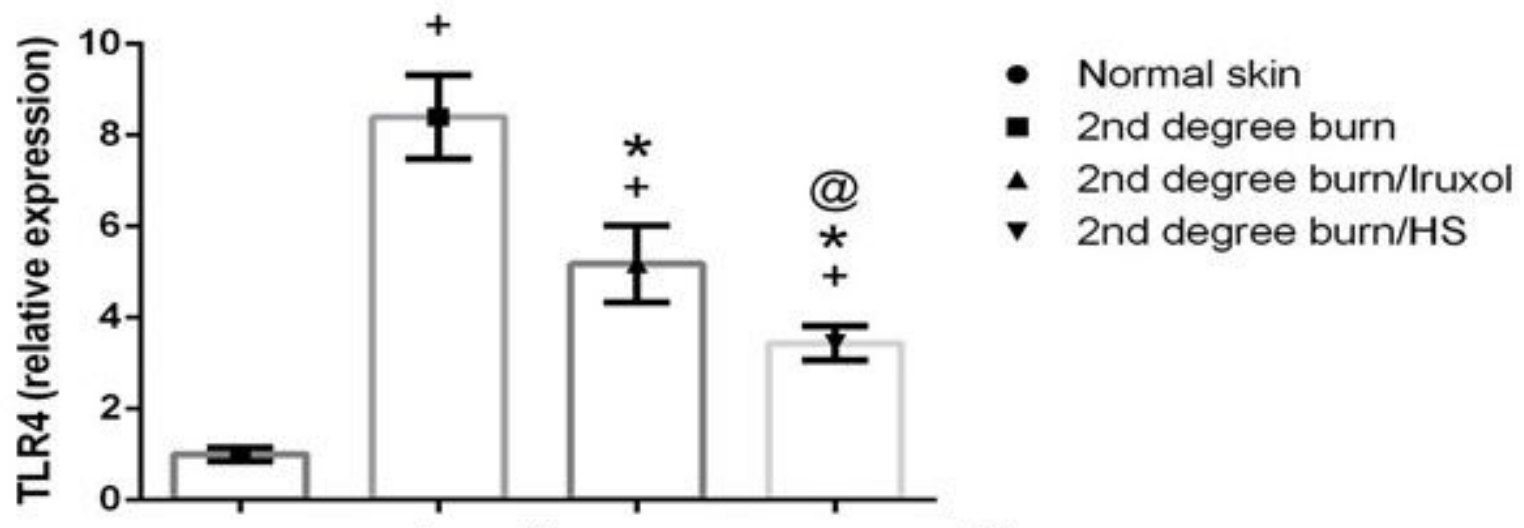

+ vs Normal skin, * vs $2^{\text {nd }}$ degree burn, @ vs $2^{\text {nd }}$ degree burn/lruxol

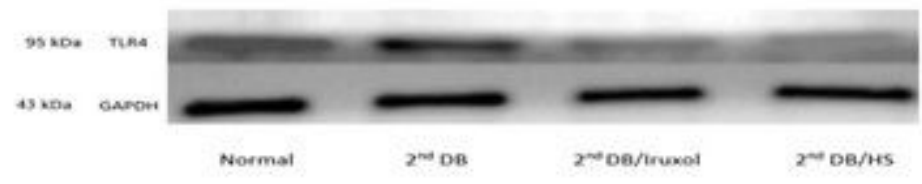

Figure 8

Effect of HS on Toll-like receptor 4 (TLR4) expression in different groups 
TLR4: Toll-like receptor 4; HS: Hibiscus sabdariffa;,$+ \mathrm{P}<0.05$ vs normal group; ${ }^{*}, \mathrm{P}<0.05 \mathrm{vs} 2$ nd degree burn group; @, $\mathrm{P}<0.05$ vs 2nd degree burn/Iruxol group. Group differences were examined by ANOVA/Tukey's Kramer multiple comparison test. $n=6$

A

PA-CI\#2
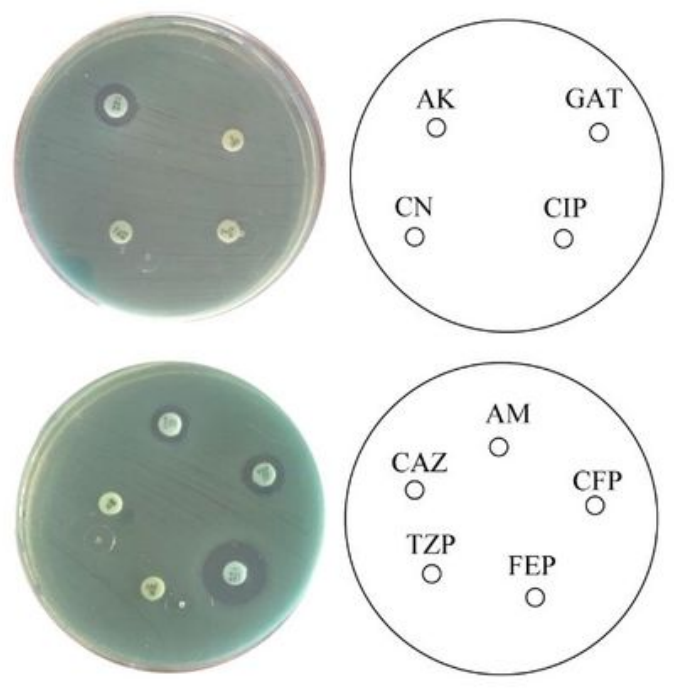

B

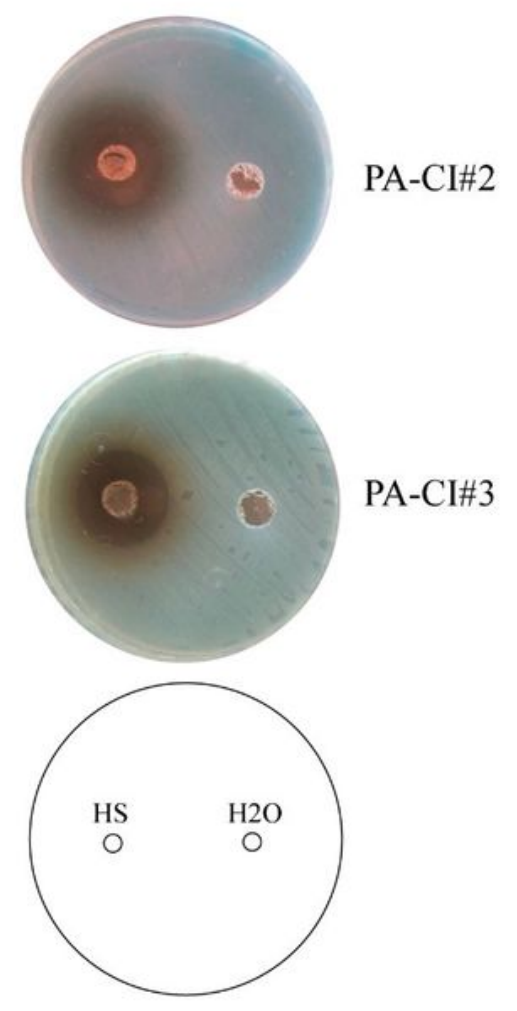

PA-CI\#3
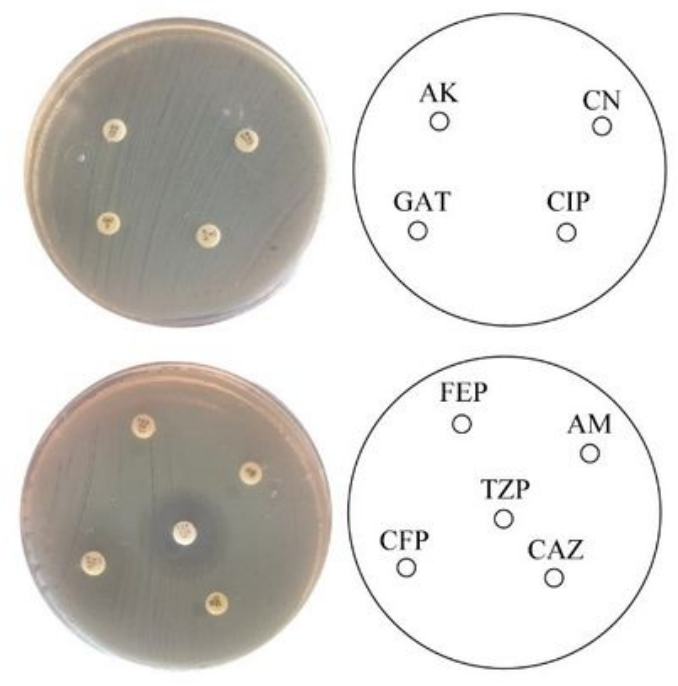

C

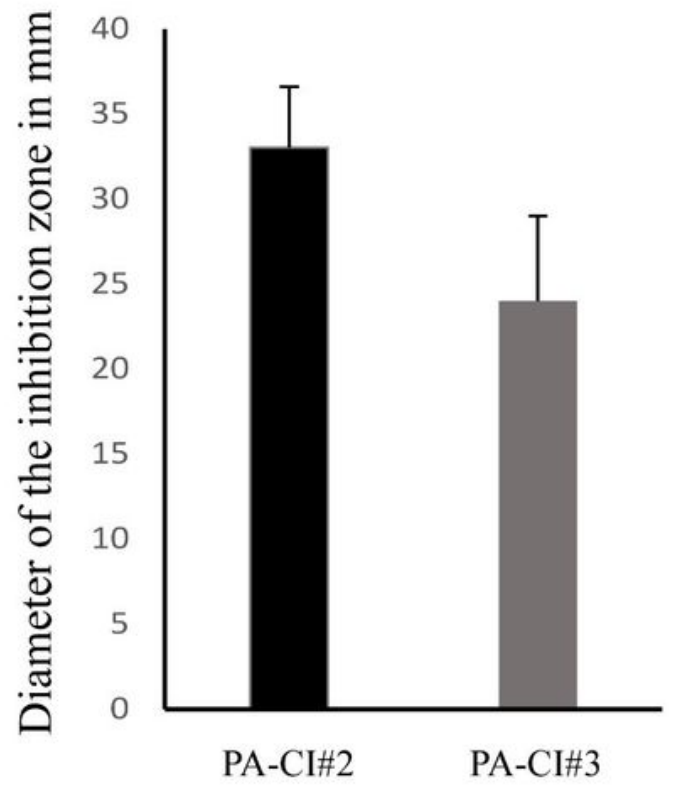

Figure 9 
A-Antibiotic sensitivity by DDM of 2 clinical isolates of $P$. aeruginosa isolated from burn wound exudates against different antibiotics (bacterial isolate was defined as multidrug resistant when resistance against 3 or more categories of antibiotics was detected. Amikacin (AK, $30 \mu \mathrm{g})$, ampicillin (AM, $10 \mu \mathrm{g})$, ceftazidime (CAZ, $30 \mu \mathrm{g})$, cefoperazone (CFP, $75 \mu \mathrm{g}$ ), ciprofloxacin (CIP, $5 \mu \mathrm{g})$, gentamicin (CN, $10 \mu \mathrm{g})$, cefepime (FEP, $30 \mu \mathrm{g})$, gatifloxacin (GAT, $5 \mu \mathrm{g}$ ), and piperacillin/tazobactam (TPZ, $110 \mu \mathrm{g})$.. B-

Bactericidal activity of HS ethanolic extract by cut-well agar diffusion against the 2 bacterial isolates in $A$. C- Diameters of the zone of inhibitions in B (the bar indicates the mean of 3 independent experiments \pm standard deviation)

\section{Supplementary Files}

This is a list of supplementary files associated with this preprint. Click to download.

- Graphicalabstract.jpg 